\title{
EDITORIAL
}

\section{Pigs might fly}

\author{
M.A. Woodhead*, A. Torres" and S. Ewig"
}

$\mathbf{T}$ here is a phrase in the English language: "pigs might fly". The phrase is used, since pigs will never fly, as a metaphor for something that will never, or is highly unlikely to ever happen. It is perhaps somewhat ironic then that although the pigs are not flying it is due to the interaction between pigs, as the source, and aviation, as the vector, that the current influenza outbreak has spread so rapidly around the world. It is reasonable to ask how this has come about and what the implications are for the respiratory community.

Three criteria must be fulfilled for a pandemic to occur. First, a novel virus, to which a significant proportion of the world population have no immunity, must evolve. Secondly, this novel virus must have the capacity to cause human disease. Thirdly, and most critically, this virus must be easily transmissible between humans. Recent experience with avian influenza viruses has shown the development of novel and virulent viruses, which although able to cause very severe disease in humans, do not have the capacity to be transmitted easily from person to person. Many will not realise that human cases of avian influenza are continuing to occur in 2009, in Indonesia, China, Viet Nam and Egypt (http://www.who.int/ csr/disease/avian_influenza/Timeline_09_03_23.pdf). However, infection is only occurring in those in direct contact with infected birds. The new H1N1 virus, that is believed to have evolved in pigs in Mexico, differs from the previous avian influenza viruses in that it is more easily transmissible between humans and this is why the current outbreak is occurring.

Influenza viruses are adapted to a number of avian and mammalian hosts and reach their greatest diversity in birds where they probably originally evolved. Most influenza viruses are host-specific, partly due to molecular interactions between the virus and host, but also because interspecies contact is unusual, in contrast to within-species contact which is common and necessary for virus transmission. The main respiratory epithelial receptor responsible for initial binding of the viral haemagglutinin is different in humans $(\alpha 2,6$ glycosidic linkage receptor) and birds ( $\alpha 2,3$ glycosidic linkage receptor), which partly explains species specificity [1]. Pigs are unusual in possessing both receptors in their respiratory epithelium. This means not only can they be infected by both human and avian viruses, but that both infections can occur

\footnotetext{
*Dept of Respiratory Medicine, Manchester Royal Infirmary, Manchester, UK. "Pulmonology Dept, Hospital Clinic of Barcelona, Institu Clinic del Torax, University of Barcelona. IDIBAPS and CIBER de Enfermedades Respiratorias (CIBERES), Barcelona, Spain. "Thoraxzentrum Ruhrgebiet, Kliniken für Pneumologie und Infektiologie, Bochum, Germany.
}

CORRESPONDENCE: M.A. Woodhead, Dept of Respiratory Medicine, Manchester Royal Infirmary, Oxford Road, Manchester, UK. E-mail: mark.woodhead@cmft.nhs.uk simultaneously. This provides the ideal situation for genetic reassortment of material from both viruses, leading to a completely novel virus.

The 1918 pandemic showed that air travel is not necessary for global virus transmission. However, such travel combines the potential for both local (by the forced incarceration of a number of individuals in a closed environment) and distant (dependent on the destination of the flight) dissemination. Indeed, in one natural experiment, $86 \%$ of those who spent more than 3 hours within a plane with one infected individual subsequently developed influenza [2]. Air travel is thus a very efficient way to produce rapid transmission over very long distances.

So what are the implications for the respiratory community? This depends on the severity of the illness and the rapidity of transmission. Neither of these factors are static. In the 1918 pandemic, the initial wave between March and July was no more serious than seasonal influenza. When the second wave began in the autumn of that year, the virus had become much more virulent [3]. At the time of writing information is incomplete. Although deaths have occurred in Mexico and the USA, most illnesses away from Mexico appear to be mild. However, by definition, most travellers are reasonably healthy. The attack rate is likely to be different in different age groups but this is yet to be determined. If the virus is then passed on to the less fit, a different pattern of illness severity may emerge. The situation may be worse in the southern hemisphere, which is just entering the winter season where cooler damper conditions favour viral transmission, and the virus may reemerge in a more virulent form later in the year.

The frontline is the national and international information services. The power of the media will, unfortunately, shape the pattern of illness presentation and any exaggeration of disease severity may promote unnecessary medical presentation, which may inadvertently increase disease transmission. Based on current knowledge of the illness being relatively mild, the media has an important job in encouraging patients who suspect that they may be infected to stay at home and seek advice remotely by phone or email.

Community physicians and accident and emergency specialists will form the main line of initial direct patient contact. In the pre-pandemic phase (World Health Organization (WHO) levels 3, 4 and 5), investigation and accurate virological diagnosis of influenza illness is important, but once level 5 is past then triage and treatment become more important. Most patients will have respiratory symptoms, usually with fever and myalgia. The majority who are not severely ill and who lack comorbidities can be managed at home with fluids, 
anti-pyretics and antivirals where appropriate. Because of its oral formulation, oseltamivir is the favoured antiviral. However, it has only been adequately tested in the nonseverely ill where its impact is modest: a reduction in fever duration of on average one day, and only if commenced within 48 hours of symptom onset [4]. Its impact in the more severely ill, particularly in patients with influenza pneumonia, is not known, although limited data suggests a benefit in those at risk [5]. A concern is the capacity for influenza A viruses to rapidly acquire oseltamivir resistance [6]. Of 1,344 isolates of influenza A (H1N1) in the second and third quarters of 2008 from the southern hemisphere, $44 \%$ were found to be resistant (http:/ / www.who.int/csr/disease/influenza/oseltamivir_summary_south_2008/en/). The few viruses tested from the current outbreak have all been oseltamivir-susceptible, while showing resistance to the adamantanes [7]. The value of this drug as a pandemic advances remains to be determined. The alternative neuraminidase inhibitor, zanamivir, is available only by the inhaled route, limiting its applicability. Whether combination of the two agents or combination with the older adamanatanes is of any advantage is not known.

Recognition of the more severely ill, including those with complications, will be important for the direction of those requiring hospital admission. No scoring tool has been prospectively tested in pandemic influenza. However, since one of the most common complications will be pneumonia, the pneumonia-specific scoring tools, CRB-65 and CURB-65, are likely to be useful in patient management. In addition to pneumonia, exacerbations of chronic obstructive pulmonary disease (COPD) are likely to be common presentations making the respiratory physician key in patient management. Management of these conditions should be according to National and International Guidelines (www.who.int). Although staphylococcal infection occurs more commonly after influenza viral infection [8], pneumococcal infection remains the most common bacterial complication overall. Initial antibiotic therapy, when indicated, should cover both organisms, with co-amoxiclav and doxycycline favoured in the non-severely ill.

Although it seems that the virulence of this microorganism is not currently very high and in most of the infected patients it only causes a mild respiratory disease, some deaths, particularly in Mexico, have been reported. The cause of these deaths is not yet known but logically they could be due to severe pulmonary complications, such as acute respiratory distress syndrome (ARDS) or secondary pneumonia. Hospitals have to be prepared to attend the most severe patients, with these complications needing admission to the intensive care unit (ICU) and artificial ventilatory support. The use of noninvasive ventilation (NIV) may be a problem, because of the potential for increased droplet transmission, and should only be undertaken with appropriate infection control measures in place. The management of these patients is usually protocolised according to local, national and international plans for influenza outbreaks and pandemics. Facing these more severe patients with influenza, we have to apply what we learnt from severe acute respiratory syndrome (SARS) [9], particularly that of a major hospital outbreak in Hong Kong with 23\% of cases that needed ICU admission and 14\% that needed mechanical ventilation. In case of ARDS, in addition to the standard ventilatory measures (lung protective ventilation), it is recommend to administer broad spectrum antibiotics such as third generation cephalosporins. Secondary pneumonia caused by Staphylococcus aureus and other pathogens, such as Streptococcus pneumoniae and Haemophilus influenzae, has to be suspected in case of persistence of fever, purulent secretions and increased levels of systemic biomarkers (procalcitonin (PCT) and C-reactive protein (CRP)). Microbiological sequential cultures of respiratory secretions may be useful to detect the initial phase of colonisation before pneumonia.

The efficacy of the coadjuvant administration of steroids is not demonstrated neither in ARDS nor in severe pneumonia, but most of the severe cases of SARS with ARDS were treated with steroids.

The isolation of patients with influenza in ICUs is a potential problem since not all units are prepared for this (isolated rooms with negative pressure). Local hospital plans have to include emergency strategies to isolate patients in the ICU to avoid nosocomial transmission [10]. The risk of transmission of the infection to personnel is very high and consequently the level of protection is the maximal recommended in the guidelines (www.who.int/csr/resources/publications/WHO_ CDS_EPR_2007_6c.pdf).

The respiratory community will be involved at every level and if the pandemic develops, usually in difficult conditions, at any one time as many as one third of staff will be absent directly due to influenza infection or the need to care for affected relatives and loved ones. At the time of writing, while occasional cases of secondary infection outside travellers from Mexico are being recorded there is no evidence of sustained community spread. It may be that this will be a useful "trial run" to prepare communities for a full-scale pandemic. This preparation will include updating pandemic planning and may most usefully include sufficient time for the development of a vaccine against the new virus. Let's hope that the pigs stay on the ground!

\section{STATEMENT OF INTEREST}

A statement of interest for M.A. Woodhead can be found at www.erj. ersjournals.com $/ \mathrm{misc} /$ statements.dtl

\section{REFERENCES}

1 Glaser L, Stevens J, Zamarin D, et al. A single amino acid substitution in 1918 influenza virus hemagglutinin changes receptor binding specificity. J Virol 2005; 79: 11533-11536.

2 Moser MR, Bender TR, Margolis HS, et al. An outbreak of influenza aboard a commercial airliner. Am J Epidemiol 1979; 110: $1-6$.

3 Potter CW. Chronicle of influenza pandemics. In: Nicholson KG, Webster RG, Hay AJ, eds. Textbook of Influenza. Oxford, Blackwell Science Ltd, 1998; pp. 3-18.

4 Jefferson T, Demicheli V, Deeks J, Rivetti D. Neuraminidase inhibitors for preventing and treating influenza in healthy adults. Cochrane Database Syst Rev 2000; CD001265.

5 Kaiser L, Wat C, Mills T, et al. Impact of oseltamivir treatment on influenza-related lower respiratory tract complications and hospitalizations. Arch Intern Med 2003; 163: 1667-1672.

6 Dharan NJ, Gubareva LV, Meyer JJ, et al. Infections with oseltamivir-resistant influenza $\mathrm{A}(\mathrm{H} 1 \mathrm{~N} 1)$ virus in the United States. JAMA 2009; 301: 1034-1041. 
7 Update: drug susceptibility of swine-origin influenza A (H1N1) viruses, April 2009. MMWR Morb Mortal Wkly Rep 2009; 58: 433435.

8 Nicholson KG. Human influenza. In: Nicholson KG, Webster RG, Hay AJ, eds. Oxford, Blackwell Science Ltd, 1998; pp. 219-266.
9 Lee N, Hui D, Wu A, et al. A major outbreak of severe acute respiratory syndrome in Hong Kong. N Engl J Med 2003; 348: 19861994.

10 Peiris JS, Yuen KY, Osterhaus AD, Stohr K. The severe acute respiratory syndrome. $N$ Engl J Med 2003; 349: 2431-2441. 